\title{
Association Between Uric Acid and Insulin-Like Growth Factor-I in Type 2 Diabetes Mellitus
}

\author{
Fang Liu' \\ Yaru Wang' \\ Qiang Zhao ${ }^{2}$ \\ Mei Zhang ${ }^{3,4}$ \\ Bo Ban $\mathbb{D}^{3,4}$ \\ 'Department of Clinical Medicine, Jining \\ Medical University, Jining, Shandong, \\ 272067, People's Republic of China; \\ ${ }^{2}$ Department of Clinical Medicine, \\ Shandong University, Jinan, Shandong, \\ 2500 I2, People's Republic of China; \\ ${ }^{3}$ Department of Endocrinology, Affiliated \\ Hospital of Jining Medical University, \\ Jining Medical University, Jining, \\ Shandong, 272029, People's Republic of \\ China; ${ }^{4}$ Chinese Research Center for \\ Behavior Medicine in Growth and \\ Development, Jining, Shandong, 272029, \\ People's Republic of China
}

Correspondence: Bo Ban Department of Endocrinology, Affiliated Hospital of Jining Medical University, Jining Medical University, 89 Guhuai Road, Jining, Shandong, 272029, People's Republic of China

Email banbo20II@163.com
Objective: This research examined the relationship between uric acid (UA) and insulin-like growth factor-1 (IGF-1) in patients with type 2 diabetes mellitus (T2DM).

Methods: This study was a cross-sectional study that included 1230 Chinese patients with T2DM. We collected general information and laboratory indicators and calculated the standard deviation score of IGF-1 (IGF-1 SDS).

Results: The univariate analysis results demonstrated a significant positive relationship between UA levels and IGF-1 SDS $(P<0.001)$. A nonlinear relationship was discovered between UA and IGF-1 SDS after adjusting for confounding factors. Multivariate piecewise linear regression showed that the levels of IGF-1 SDS increased when UA was $<4.17 \mathrm{mg} / \mathrm{dl}$ ( $\beta 0.12,95 \%$ CI $-0.04,0.28 ; P=0.121)$ and decreased when UA was $>4.17 \mathrm{mg} / \mathrm{dl}(\beta$ $-0.38,95 \%$ CI $-0.64,-0.12 ; P=0.004)$.

Conclusion: This study showed a nonlinear relationship between UA and IGF-1 in Chinese adults with T2DM. When the UA levels reached the inflection point, IGF-1 levels negatively correlated with the increase in UA. Further studies are needed to explore this relationship and mechanisms in the future.

Keywords: uric acid, insulin-like growth factor-1, type 2 diabetes mellitus

The incidence of diabetes is increasing worldwide. An estimated 463 million people had diabetes worldwide in 2019, and this rate is expected to rise to 578 million in 2030. Type 2 diabetes mellitus (T2DM) accounts for approximately $90 \%$ of all cases of diabetes, and the prevalence of macrovascular and microvascular diseases is relatively high. ${ }^{1}$

Insulin-like growth factor-1 (IGF-1) is a pluripotent growth factor that plays an important role in tissue energy metabolism and growth. ${ }^{2}$ Similar to insulin, IGF-1 increases insulin sensitivity and peripheral glucose uptake, which reduce liver glucose production. ${ }^{3}$ Low IGF-1 levels are associated with an increased incidence and all-cause mortality in men and women with $\mathrm{T} 2 \mathrm{DM},{ }^{4}$ and insulin sensitivity is increased by injecting IGF-1 into patients with T2DM or severe insulin resistance. ${ }^{5}$ The growth hormone-IGF-1 axis also plays a potential role in the pathogenesis of diabetes-related complications, and low levels of IGF-1 contribute to the development of diabetic complications, including diabetic neuropathy, diabetic nephropathy, and diabetic retinopathy. ${ }^{6}$ Maintaining circulating IGF-1 levels is of great significance for preventing the occurrence and development of T2DM. Therefore, it is important to explore the influencing factors of IGF-1 in patients with T2DM. The level of IGF-1 is closely related to the nutritional status of the body and diet quality and quantity. Inadequate nutrition intake has a negative effect on the 
biological activity of IGF-1. ${ }^{7}$ When energy is scarce, plasma IGF-1 levels are significantly reduced, and increased calorie intake increases the IGF-1 level. ${ }^{8}$

Uric acid (UA) is the end product of nucleic acid purine metabolism. The kidney is an important organ for UA metabolism. Most UA is freely filtered in the kidneys, and approximately $90 \%$ of UA is reabsorbed by nephrons. ${ }^{9}$ $\mathrm{UA}$ is a redox agent that promotes and resists oxidation, and it has important physiological effects in humans. ${ }^{10}$ Serum UA levels are independently associated with an increased risk of diabetic peripheral neuropathy in T2DM patients, ${ }^{11}$ and it may predict the development of diabetic retinopathy and diabetic nephropathy. ${ }^{12,13} \mathrm{UA}$ levels are related to dietary factors and reflect dietary intake to a certain extent. ${ }^{14}$ Potential pathophysiology and prescribed dietary patterns lead to nutritional deficiency and imbalance in T2DM patients. Previous studies showed that hospitalized T2DM patients had poorer nutritional status than nondiabetic patients, which may affect UA levels. ${ }^{15}$

Recent studies found that an increase in serum UA levels was closely related to the occurrence of metabolic syndromes other than gout, such as dyslipidaemia, abnormal glucose tolerance, hypertension, central obesity and other metabolic disorders. ${ }^{16}$ UA also induces insulin resistance via endothelial cell mitochondrial oxidative stress. ${ }^{17}$ The relationship between UA and IGF-1 has received some attention, but there is little research on this relationship. Wang et al found a nonlinear relationship between IGF-1 and UA levels in Chinese children and adolescents with idiopathic short stature, and high and low levels of UA had adverse effects on IGF-1. ${ }^{18}$ Sesti et al demonstrated a negative correlation between IGF-1 and UA levels in nondiabetic adults. ${ }^{19}$

However, the relationship between UA and IGF-1 was not studied in patients with T2DM. Because UA and IGF1 are associated with diabetic complications, exploring the relationship between UA and IGF-1 in T2DM patients will improve our understanding of the complications of T2DM and may provide new insights on its occurrence and development mechanism. Therefore, this study examined the relationship between IGF-1 and UA in patients with T2DM in China.

\section{Subjects and Methods Study Population}

This cross-sectional study analysed data collected from 1304 participants from the Department of Endocrinology,
Affiliated Hospital of Jining Medical University between July 1, 2017 and June 30, 2019. The diagnosis of T2DM referred to the diagnosis and classification standards of diabetes proposed by the World Health Organization in 1999: diabetes symptoms + plasma glucose level at any time of $11.1 \mathrm{mmol} / \mathrm{l}(200 \mathrm{mg} / \mathrm{dl})$, fasting blood glucose (FPG) levels of $7.0 \mathrm{mmol} / \mathrm{l}(126 \mathrm{mg} / \mathrm{dl})$, or 2-h plasma glucose levels of $11.1 \mathrm{mmol} / \mathrm{l}(200 \mathrm{mg} / \mathrm{dl})$ in an oral glucose tolerance test. ${ }^{20}$ All of the subjects enrolled in the study were diagnosed with T2DM based on the 1999 WHO standards, had a history of diabetes diagnosis, or currently used oral hypoglycaemics or insulin. Subjects with serious diseases of the heart, brain, liver, kidney, or blood, with tumours, without acute complications, such as diabetic ketoacidosis or severe infection in the near future, were taking medicine for gout, or who lacked data on IGF1 and UA levels were excluded. This study eventually enrolled 1230 patients according to these criteria.

The Human Ethics Committee of the Affiliated Hospital of Jining Medical College (Shandong, China) approved the study. All patients were informed of the aims of the study and signed informed consent forms.

\section{General Information}

General information of the participants was obtained from hospital medical records, including sex, age, duration of diabetes, family history of diabetes and use of hypoglycaemic drugs. There were eight types of hypoglycaemic drugs: sulfonylurea, glinide, biguanide, thiazolidinedione, alpha glucosidase inhibitor, dipeptidyl peptidase-4 (DPP4) inhibitors, sodium-glucose cotransporter-2 (SGTL2) inhibitors, and insulin.

\section{Anthropomorphic Measurements}

Participants' height was measured after removal of hats and shoes. All participants were measured using the same height metre (Nantong Best Industrial Co. Ltd., Jiangsu, China) with an allowable error range of $0.1 \mathrm{~cm}$. Weights were measured under fasting conditions using the same scale (China Jiangsu Wuxi Scale Factory Co., Ltd.) to the nearest $0.1 \mathrm{~kg}$. Body mass index (BMI) was calculated as weight (kilograms)/height (metres squared). A trained nurse measured waist circumference midway between the costal margin and iliac crest, and the hip circumference was measured at the widest point around the greater trochanter. The waist-to-hip ratio (WHR) was calculated as the waist circumference (centimetre)/hip circumference (centimetre). 


\section{Laboratory Measurements}

Following an 8-12-h period of fasting, fasting blood samples were obtained from all participants for laboratory analyses. Serum IGF-1 concentrations were estimated based on a chemiluminescence assay (DPC IMMULITE 1000 analyser, SIEMENS, Germany) with intra-assay and inter-assay coefficients of variation of 3.0 and $6.2 \%$, respectively. Total cholesterol (TC), high-density lipoprotein cholesterol (HDL-C), lowdensity lipoprotein cholesterol (LDL-C), triglyceride (TG), fasting plasma glucose(FPG), and UA levels were determined using an auto biochemical analyser (Cobas c702, Roche; Shanghai, China). Glycated haemoglobin (HbAlc) concentration was measured using a high-performance liquid chromatography method. Plasma glucose was measured using a hexokinase method. Plasma insulin concentrations were determined using a chemiluminescent immunoassay.

Insulin resistance (IR) was estimated by homeostasis model assessment for insulin resistance (HOMA-IR) = FPG $(\mathrm{mmol} / \mathrm{L}) \times$ fasting plasma insulin $(\mathrm{FINS})(\mu \mathrm{IU} / \mathrm{mL}) /$ 22.5. The standard deviation score of IGF-1 (IGF-1 SDS) was calculated according to a previous study. ${ }^{21}$

\section{Statistical Analysis}

All statistical analyses were performed using Empower Stats (www.empowerstats.com, X\&Y solutions, Inc. Boston MA) and R software version 3.6.1 (http://www.r-project.org). We initially described the general information and biochemical variables of the subjects (Table 1). We express continuous variables with a normal distribution as means (standard deviation) and continuous variables with a non-normal distribution as medians (quartile). Categorical variables are expressed as frequencies or percentages. A univariate analysis model (Table 2) was used to examine whether UA and other anthropometrical and biochemical variables correlated with IGF-1 SDS. We examined the relationship between UA and IGF-1 SDS using smooth curve fitting after adjustment for potential confounders (Figure 1). We further used a multivariate piecewise linear regression model to assess independent correlations between UA and IGF-1 SDS according to smooth curve fitting (Table 3). Two-sided $\mathrm{p}$ values $<0.05$ were regarded as significant differences.

\section{Results}

\section{Clinical and Laboratory Characteristics of the Subjects}

The clinical and laboratory characteristics of the subjects are shown in Table 1. A total of $575(46.75 \%)$ males and
Table I Baseline Characteristics of Patients with Type 2 Diabetes Mellitus

\begin{tabular}{|l|l|}
\hline Variables & All \\
\hline Number & 1230 \\
Sex (male, $n, \%)$ & $575(46.75 \%)$ \\
Age (years) & $54.18 \pm 11.89$ \\
Duration of disease(years) & $8.47 \pm 6.90$ \\
Diabetes family history (n,\%) & $651(52.93 \%)$ \\
BMI (kg/m²) & $26.16 \pm 3.50$ \\
WHR & $0.93 \pm 0.26$ \\
TC (mmol/L) & $4.45 \pm 1.18$ \\
TG (mmol/L) & $1.32(0.95-1.80)$ \\
HDL-C (mmol/L) & $1.11 \pm 0.28$ \\
LDL-C (mmol/L) & $2.82 \pm 0.58$ \\
BUN ( $\mu$ mol/L) & $5.73 \pm 4.15$ \\
Cr ( $\mu$ mol/L) & $57.82 \pm 15.43$ \\
UA (mg/dl) & $4.71 \pm 1.36$ \\
IGF-I (ng/mL) & $158.76 \pm 63.14$ \\
IGF-I SDS & $0.26 \pm 1.42$ \\
FPG (mmol/L) & $9.49 \pm 4.03$ \\
HbAIc (\%) & $8.88 \pm 1.91$ \\
HOMA-IR & $2.37(1.52-3.94)$ \\
\hline Hypoglycaemic drugs & \\
Sulfonylurea (n,\%) & $481(39.11 \%)$ \\
Glinide (n,\%) & $81(6.59 \%)$ \\
Biguanide (n,\%) & $571(46.42 \%)$ \\
Thiazolidinedione (n,\%) & $40(3.25 \%)$ \\
Alpha glucosidase inhibitor (n,\%) & $409(33.25 \%)$ \\
DPP-4 inhibitors (n,\%) & $93(7.56 \%)$ \\
SGTL2 inhibitors (n,\%) & $3(0.24 \%)$ \\
Insulin (n,\%) & $408(33.17 \%)$ \\
\hline
\end{tabular}

Note: Data was presented as mean \pm standard deviation, median (interquartile range) and number (percentage).

Abbreviations: BMI, body mass index; WHR, waist-to-hip ratio; IGF-I, insulin-like growth factor-I; IGF-I SDS, insulin-like growth factor-I standard deviation score; TC, total cholesterol; TG, triglyceride; HDL-C, high-density lipoprotein cholesterol; LDL-C, low-density lipoprotein cholesterol; BUN, blood urea nitrogen; $\mathrm{Cr}$, creatinine; UA, uric acid; FPG, fasting plasma glucose; HbAlc, glycated hemoglobin; HOMA-IR, homeostasis model assessment for insulin resistance.

$655(53.25 \%)$ females were included in this research. The mean age of the participants was $54.18 \pm 11.89$ years. They had an average duration of $8.51 \pm 6.74$ years of disease. The mean levels of IGF-1 SDS and UA were $0.26 \pm 1.42$ and $4.71 \pm 1.36 \mathrm{mg} / \mathrm{dl}$, respectively.

\section{Correlations Between IGF-I SDS and Anthropometrical and Biochemical Variables}

Univariate linear regression analysis was performed to determine the relationships between clinical parameters and IGF-1 SDS. As shown in Table 2, we observed significant positive 
Table 2 Univariate Analysis of Factors Affecting IGF-I SDS

\begin{tabular}{|c|c|c|}
\hline Covariate & $\beta(95 \% \mathrm{Cl})$ & $P$-value \\
\hline UA (mg/dl) & $0.01(-0.02,0.03)$ & $<0.001$ \\
\hline \multicolumn{3}{|l|}{ Sex } \\
\hline Male & Reference & \\
\hline Female & $0.42(-0.37,1.21)$ & 0.294 \\
\hline Age (years) & $-0.04(-0.07,-0.0 \mathrm{I})$ & 0.031 \\
\hline Duration of disease(years) & $-1.6(-2.3,-1.0)$ & $<0.001$ \\
\hline \multicolumn{3}{|l|}{ Diabetes family history } \\
\hline Yes & Reference & \\
\hline No & $-0.68(-1.47,0.10)$ & 0.089 \\
\hline BMI $\left(\mathrm{kg} / \mathrm{m}^{2}\right)$ & $0.08(-0.03,0.19)$ & 0.147 \\
\hline WHR & $-0.01(-1.54,1.52)$ & 0.991 \\
\hline $\mathrm{TC}(\mathrm{mmol} / \mathrm{L})$ & $-0.01(-0.09,0.07)$ & 0.867 \\
\hline TG (mmol/L) & $-0.03(-0.07,0.01)$ & 0.086 \\
\hline $\mathrm{HDL}-\mathrm{C}(\mathrm{mmol} / \mathrm{L})$ & $0.10(0.06,0.14)$ & $<0.001$ \\
\hline LDL-C (mmol/L) & $-0.01(-0.07,0.05)$ & 0.822 \\
\hline $\mathrm{BUN}(\mu \mathrm{mol} / \mathrm{L})$ & $0.05(-0.04,0.15)$ & 0.286 \\
\hline $\mathrm{Cr}(\mu \mathrm{mol} / \mathrm{L})$ & $0.01(-0.02,0.03)$ & 0.551 \\
\hline FBG (mmol/L) & $0.37(0.27,0.46)$ & $<0.001$ \\
\hline $\mathrm{HbAlc}(\%)$ & $-0.08(-0.13,-0.02)$ & $<0.001$ \\
\hline HOMA-IR & $-0.13(-0.19,-0.07)$ & $<0.001$ \\
\hline
\end{tabular}

Note: Significant results $(P<0.05)$ are indicated in bold.

Abbreviations: $\mathrm{Cl}$, confidence interval; $\mathrm{BMI}$, body mass index; WHR, waist-to-hip ratio; IGF-I, insulin-like growth factor-I; IGF-I SDS, insulin-like growth factor-I standard deviation score; TC, total cholesterol; TG, triglyceride; HDL-C, highdensity lipoprotein cholesterol; LDL-C, low-density lipoprotein cholesterol; BUN, blood urea nitrogen; $\mathrm{Cr}$, creatinine; UA, uric acid; FPG, fasting plasma glucose; HbAIc, glycated hemoglobin; HOMA-IR, homeostasis model assessment for insulin resistance.

correlations between IGF-1 SDS and UA, HDL-C, and FPG $(\mathrm{p}<0.01)$ and negative correlations between IGF-1 SDS and age, duration of disease, HbA1c and HOMA-IR $(p<0.01)$. There was no significant correlation between IGF-1 SDS and sex, BMI, WHR, family history, BUN or $\mathrm{Cr}(\mathrm{p}>0.05)$.

\section{Non-Linear Relationship Between UA and IGF-I SDS}

As shown in Figure 1, smooth curve fitting showed a nonlinear relationship between UA and IGF-1 SDS after adjusting for sex, age, duration of disease, BMI, TC, TG, HDL-C, LDL-C, Cr, FPG, HbA1c, HOMA-IR and hypoglycaemic drugs. There was a two-stage change and a breakpoint in this curve. When the UA level was less than the breakpoint, there was a positive relationship between UA and IGF-1 SDS. When the UA was greater than the breakpoint, there was a negative relationship between UA and IGF-1 SDS. As shown in Table 3, we further analysed the threshold effect based on curve fitting, and the data indicated that the inflection point of UA was $4.17 \mathrm{mg} / \mathrm{dl}$. IGF-1 SDS levels increased as UA increased when UA was less than $4.17 \mathrm{mg} / \mathrm{dl}(\beta 0.12,95 \% \mathrm{CI}-0.04,0.28 ; P=0.121)$. However, there was no statistical significance. IGF-1 levels decreased as UA increased when UA was greater than $4.17 \mathrm{mg} / \mathrm{dl}(\beta-0.38,95 \% \mathrm{CI}-0.64,-0.12 ; P=0.004)$.

\section{Discussion}

This cross-sectional study observed a nonlinear relationship between UA and IGF-1 SDS in patients with T2DM, and the UA inflection point was $4.17 \mathrm{mg} / \mathrm{dl}$. When UA was greater than $4.17 \mathrm{mg} / \mathrm{dl}$, the negative relationship between UA and IGF-1 SDS was significant.

Hyperuricaemia is common in patients with T2DM, with a prevalence of $22 \%{ }^{22}$ The standard of hyperuricemia is defined by UA levels above $6 \mathrm{mg} / \mathrm{dl}$ in female and above $7 \mathrm{mg} / \mathrm{dl}$ in male. ${ }^{23}$ According to this criterion, hyperuricaemia was present in $7.24 \%$ of patients with diabetes in our study. We speculate that the differences in our study were related to several factors, such as the hyperuricaemia criterion, environment, dietary structure, and ethnic heterogeneity. Insulin resistance is an important feature in T2DM patients. The reason for the elevated UA levels in diabetic patients may be related to insulin resistance. Insulin resistance leads to hyperuricaemia by increasing the sodium-hydrogen ion exchange of renal tubules and the secretion of hydrogen ions, which promote the reabsorption of UA. ${ }^{24}$ Diabetic patients have many poor dietary habits, such as a high glucose and high fat diet, ${ }^{25}$ which may also lead to an increase in UA levels. These factors may contribute to the elevated UA levels in the diabetic population.

Previous research on the relationship between UA and IGF-1 primarily concentrated on healthy adults and adolescent populations, and no research examined their relationship in type 2 diabetes. Our study found a negative correlation between UA and IGF-1 only when $\mathrm{UA}>4.17 \mathrm{mg} / \mathrm{dl}$ in people with T2DM. This trend is consistent with findings in nondiabetic individuals. ${ }^{19}$ Wang et al found that IGF-1 levels increased with increasing UA levels when UA was $>301 \mu \mathrm{mol} / 1$ in children with idiopathic short stature, ${ }^{18}$ which was inconsistent with the 


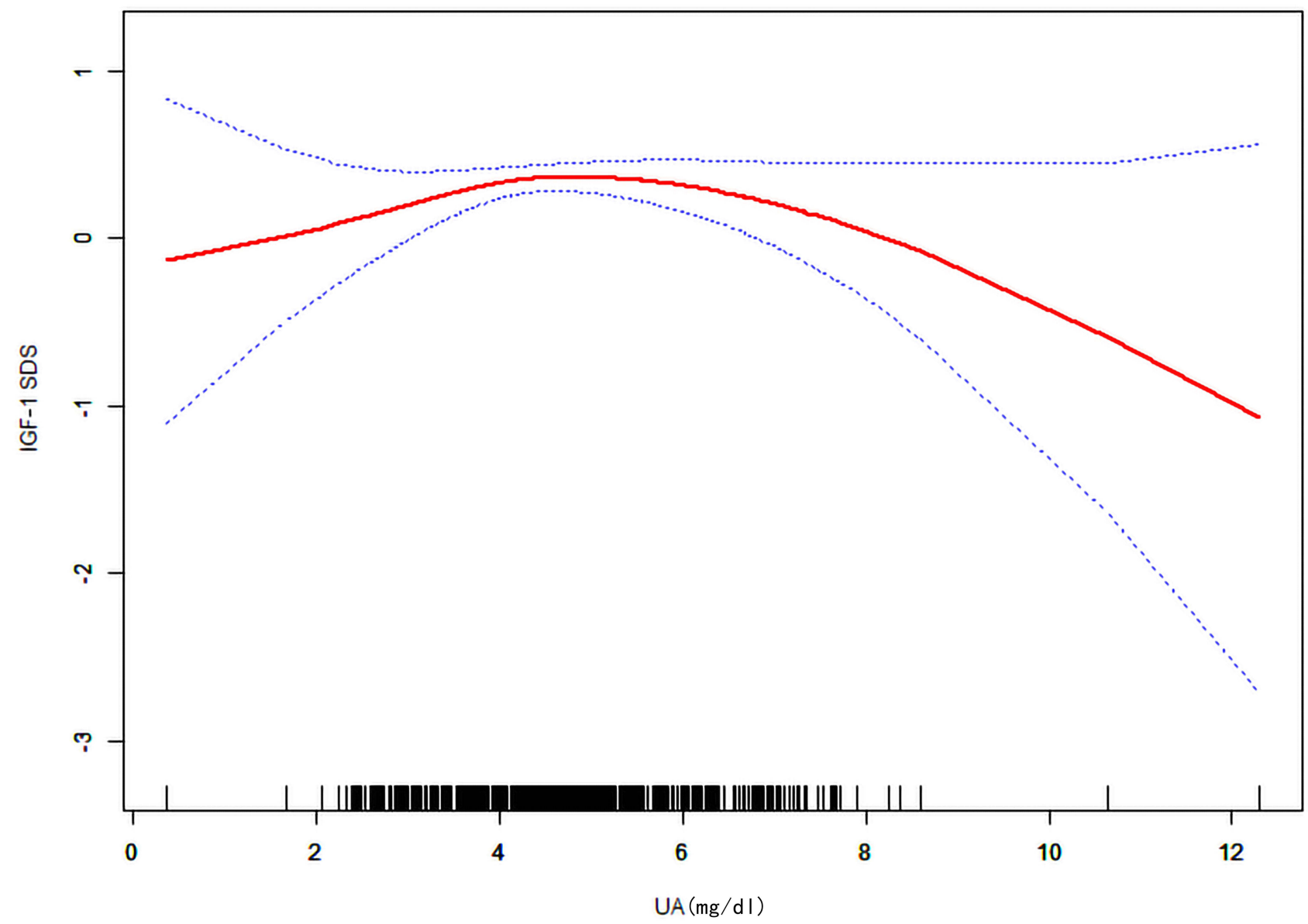

Figure I The relationship between UA and IGF-I SDS. A threshold, nonlinear association between UA and IGF-I SDS was found in a generalized additive model (GAM). Solid red line represents the smooth curve fit between variables. Dotted line represents the $95 \%$ of confidence interval from the fit. All adjusted for sex, age, duration of disease, BMI, TC, TG, HDL-C, LDL-C, Cr, FPG, HbAIc, HOMA-IR, hypoglycemic drugs.

UA inflection point in diabetic patients and may be due to the different dietary habits of adults and children. We also found that IGF-1 levels decreased with increasing UA levels when UA reached the inflection point, but this turning point was within the normal range and did not meet the criteria for hyperuricaemia. This result suggests that even in the absence of hyperuricaemia in the diabetic population, UA above a certain level within the normal range adversely affects IGF-1. The reason why the increased UA reduces IGF-1 levels may be related to the effect of UA on IGF-1 expression in the liver. Growth hormone stimulation of the Janus kinase-2 (JAK2)-signal transducer and activator of transcription-5 (STAT5) signalling pathway is an important regulatory pathway of IGF-1 gene expression. Human liver cancer cell exposure to UA induced oxidative stress and protein translation that weakened the conduction ability of the pathway and downregulated IGF-1 gene expression. ${ }^{19}$ Insulin resistance is another important factor that affects the relationship between UA and IGF-1. There is a close relationship between insulin resistance and high levels of UA. ${ }^{24}$ Increased UA is deposited in the pancreas, which leads to further damage to islet $\beta$ cell function and

Table 3 The Independent Association Between UA and IGF-I SDS by Multivariate Piecewise Linear Regression

\begin{tabular}{|l|l|l|l|}
\hline Inflection Point of UA(mg/d) & Effect Size $(\boldsymbol{\beta})$ & $\mathbf{9 5 \%} \mathbf{C l}$ & $\boldsymbol{P}$-value \\
\hline$<4.17$ & 0.12 & $(-0.04,0.28)$ & 0.121 \\
\hline$\geq 4.17$ & -0.38 & $(-0.64,-0.12)$ & $\mathbf{0 . 0 0 4}$ \\
\hline
\end{tabular}

Notes: Significant results $(\mathrm{P}<0.05)$ are indicated in bold. Adjusted: sex, age, duration of disease, BMI, TC, TG, HDL-C, LDL-C, Cr, FPG, HbAIc, HOMA-IR, hypoglycemic drugs. 
aggravation of insulin resistance via the induction of cytotoxicity and apoptosis of $\beta$ cells. ${ }^{26}$ Elevated UA levels also inhibit insulin signalling pathways, which directly leads to insulin resistance. ${ }^{27}$ Diabetic patients with insulin resistance exhibit lower circulating IGF-1 levels due to a decrease in growth hormone reactivity. ${ }^{28}$

Our study has some limitations. First, this study is a cross-sectional study that only showed a nonlinear relationship between UA and IGF-1 levels, and it could not determine a causal relationship, which requires verification by prospective studies. Second, our study was performed in patients with diagnosed diabetes, and the results cannot be extrapolated to the newly diagnosed diabetic population. And all of our patients were inpatients, so the results cannot be generalized to general T2DM patients. Third, we did not measure IGF-binding protein (IGF-BP) or growth hormone.

\section{Conclusion}

The present study described a nonlinear relationship between UA and IGF-1 SDS in patients with T2DM after adjusting for potential confounding factors. However, the causal relationship between the two factors is not certain, and further research is needed to identify the possible mechanisms.

\section{Abbreviations}

BMI, body mass index; WHR, waist hip ratio; IGF-1, insulin-like growth factor-1; IGF-1 SDS, insulin-like growth factor-1 standard deviation score; TC, total cholesterol; TG, triglyceride; HDL-C, high-density lipoprotein cholesterol; LDL-C, low-density lipoprotein cholesterol; BUN, blood urea nitrogen; $\mathrm{Cr}$, creatinine; UA, uric acid; FPG, fasting plasma glucose; HbAlc, glycated hemoglobin; HOMA-IR, homeostasis model assessment for insulin resistance.

\section{Data Sharing Statement}

The datasets used and/or analysed in the current study are available from the corresponding authors upon reasonable request.

\section{Ethics Approval and Consent to Participate}

Human Research Committee Affiliated Hospital of Jining Medical University approved the study. All procedures were performed in accordance with ethical standards laid out in the Declaration of Helsinki. All of the patients were informed of the aims of the study, and written informed consent was obtained.

\section{Consent for Publication}

All authors have read and approved the content, and they agree to submit it for consideration for publication in the journal.

\section{Disclosure}

The authors declare that they have no conflicts of interest.

\section{References}

1. Saeedi P, Petersohn I, Salpea P, et al. Global and regional diabetes prevalence estimates for 2019 and projections for 2030 and 2045: results from the international diabetes federation diabetes atlas, 9th edition. Diabetes Res Clin Pract. 2019;157:107843. doi:10.1016/j. diabres.2019.107843

2. Aguirre GA, De Ita JR, de la Garza RG, et al. Insulin-like growth factor-1 deficiency and metabolic syndrome. $J$ Transl Med. 2016;14:3. doi:10.1186/s12967-015-0762-z

3. Boulware SD, Tamborlane WV, Rennert NJ, et al. Comparison of the metabolic effects of recombinant human insulin-like growth factor-I and insulin. Dose-response relationships in healthy young and middle-aged adults. J Clin Invest. 1994;93(3):1131-1139. doi:10.1172/JCI117065

4. Miyake H, Kanazawa I, Sugimoto T. Decreased serum insulin-like growth factor-I level is associated with the increased mortality in type 2 diabetes mellitus. Endocr J. 2016;63(9):811-818. doi:10.1507/ endocrj.EJ16-0076

5. Moses AC, Young SC, Morrow LA, et al. Recombinant human insulin-like growth factor I increases insulin sensitivity and improves glycemic control in type II diabetes. Diabetes. 1996;45(1):91-100. doi:10.2337/diab.45.1.91

6. Thrailkill KM. Insulin-like growth factor-I in diabetes mellitus: its physiology, metabolic effects, and potential clinical utility. Diabetes Technol Ther. 2000;2(1):69-80. doi:10.1089/152091599316775

7. Maggio M, De Vita F, Lauretani F, et al. IGF-1, the cross road of the nutritional, inflammatory and hormonal pathways to frailty. Nutrients. 2013;5(10):4184-4205. doi:10.3390/nu5104184

8. Underwood LE, Clemmons DR, Maes M, et al. Regulation of somatomedin-C/insulin-like growth factor I by nutrients. Horm Res. 1986;24(2-3):166-176. doi:10.1159/000180556

9. Bobulescu IA, Moe OW. Renal transport of uric acid: evolving concepts and uncertainties. Adv Chronic Kidney Dis. 2012;19 (6):358-371. doi:10.1371/journal.pone.0078284

10. Hayden MR, Tyagi SC. Uric acid: a new look at an old risk marker for cardiovascular disease, metabolic syndrome, and type 2 diabetes mellitus: the urate redox shuttle. Nutr Metab. 2004;1(1):10. doi:10.1186/1743-7075-1-10

11. Yu S, Chen Y, Hou X, et al. Serum uric acid levels and diabetic peripheral neuropathy in type 2 diabetes: a systematic review and meta-analysis. Mol Neurobiol. 2016;53(2):1045-1051. doi:10.1007/ s12035-014-9075-0

12. Hou L, Shi Y, Wang S, et al. Associations of serum uric acid level with diabetic retinopathy and albuminuria in patients with type 2 diabetes mellitus. J Int Med Res. 2020;48(12):1220763532. doi:10.1177/0300060520963980

13. De Cosmo S, Viazzi F, Pacilli A, et al. Serum uric acid and risk of CKD in type 2 diabetes. Clin J Am Soc Nephrol. 2015;10 (11):1921-1929. doi:10.2215/CJN.03140315 
14. Beydoun MA, Fanelli-Kuczmarski MT, Canas J-A, et al. Dietary factors are associated with serum uric acid trajectory differentially by race among urban adults. Br $J$ Nutr. 2018;120(8):935-945. doi: $10.1017 /$ S0007114518002118

15. Serrano VC, Lopez GJ, Garcia CS, et al. Influence of nutritional status on hospital length of stay in patients with type 2 diabetes. Endocrinol Diabetes Nutr. 2020;67(10):617-624. doi:10.1016/j. endinu.2020.05.004

16. Ni W, Wang R, Liu Z, et al. Association of serum uric acid with metabolic syndrome and its components: a Cross-Sectional Study in Chinese Coastal Population. Metab Syndr Relat Disord. 2020;18 (2):103-109. doi:10.1089/met.2019.0043

17. King C, Lanaspa MA, Jensen T, et al. Uric acid as a cause of the metabolic syndrome. Contrib Nephrol. 2018;192:88-102. doi:10.1159/000484283

18. Wang P, Ji B, Shao Q, et al. Association between insulin-like growth factor-1 and uric acid in Chinese children and adolescents with idiopathic short stature: a Cross-Sectional Study. Biomed Res Int. 2018;2018:4259098. doi:10.1155/2018/4259098

19. Sesti G, Hribal ML, Procopio T, et al. Low circulating insulin-like growth factor-1 levels are associated with high serum uric acid in nondiabetic adult subjects. Nutr Metab Cardiovasc Dis. 2014;24 (12):1365-1372. doi:10.1016/j.numecd.2014.06.012

20. Grimaldi A, Heurtier A. Diagnostic criteria for type 2 diabetes. Rev Prat. 1999;49(1):16-21.

21. Isojima T, Shimatsu A, Yokoya S, et al. Standardized centile curves and reference intervals of serum insulin-like growth factor-I (IGF-I) levels in a normal Japanese population using the LMS method. Endocr J. 2012;59(9):771-780. doi:10.1507/endocrj.ej12-0110
22. Arersa KK, Wondimnew T, Welde M, et al. Prevalence and determinants of hyperuricemia in type 2 diabetes mellitus patients attending Jimma Medical Center, Southwestern Ethiopia, 2019. Diabetes Metab Syndr Obes. 2020;13:2059-2067. doi:10.2147/DMSO. $\mathrm{S} 252825$

23. Multidisciplinary Expert Task Force on Hyperuricemia and Related Diseases. Chinese multidisciplinary expert consensus on the diagnosis and treatment of hyperuricemia and related diseases. Chin Med J. 2017;130(20):2473-2488. doi:10.4103/0366-6999.216416

24. Facchini F. Relationship between resistance to insulin-mediated glucose uptake, urinary uric acid clearance, and plasma uric acid concentration. JAMA. 1991;266(21):3008-3011. doi:10.1001/ jama.1991.03470210076036

25. Purifine AS, Ekou FK, Konan YE, et al. Dietary habits among type 2 diabetic patients attending the Abidjan Diabetes Centre. Sante Publique (Paris). 2017;29(3):423-430.

26. Jia L, Xing J, Ding $Y$, et al. Hyperuricemia causes pancreatic beta-cell death and dysfunction through NF-kappaB signaling pathway. PLoS One. 2013;8(10):e78284. doi:10.1371/journal. pone. 0078284

27. Zhu Y, Hu Y, Huang T, et al. High uric acid directly inhibits insulin signalling and induces insulin resistance. Biochem Biophys Res Commun. 2014;447(4):707-714. doi:10.1016/j.bbrc.2014.04.080

28. Ji S, Guan R, Frank SJ, et al. Insulin inhibits growth hormone signaling via the growth hormone receptor/JAK2/STAT5B pathway. J Biol Chem. 1999;274(19):13434-13442. doi:10.1074/ jbc.274.19.13434
International Journal of General Medicine

\section{Publish your work in this journal}

The International Journal of General Medicine is an international, peer-reviewed open-access journal that focuses on general and internal medicine, pathogenesis, epidemiology, diagnosis, monitoring and treatment protocols. The journal is characterized by the rapid reporting of reviews, original research and clinical studies

\section{Dovepress}

across all disease areas. The manuscript management system is completely online and includes a very quick and fair peer-review system, which is all easy to use. Visit http://www.dovepress.com/ testimonials.php to read real quotes from published authors. 